\title{
SOLID-STATE MICROWAVE PROCESSOR FOR FOOD TREATMENT
}

\author{
M.Fiore $^{1}$, N. Di Modugno ${ }^{2}$, F. Pellegrini ${ }^{3}$, M. Roselli ${ }^{4}$ \\ ${ }^{1,2}$ LEANFA Srl, Via C. A. Dalla Chiesa 6, Ruvo di Puglia, Italy \\ marco.fiore@leanfa.com,nicola.dimodugno@leanfa.com \\ ${ }^{3,4}$ Sergio Fontana Srl, Via Moscatello 2a trav., 76012 -Canosa, Italy \\ f.pellegrini@fontanasergio.it,m.roselli@fontanasergio.it
}

Keywords: microwave, solid-state, self-regulation, software, adaptive

\section{Introduction}

In a global context, the Food Processing (FP) industry is a leading manufacturing sector. Even in the periods of economic downturn, FP industry keeps being a stable, non-cyclical and robust sector with a steady growth in turnover and profitability. The popularity and sales of processed foods are still increasing, not only in the high-income Countries but even more in developing, low-medium income ones. Challenges in the market are dependent on the booming growth population, projected to hit the peak of 9Bn people by 2050: as a result, raw materials as well as natural resources will come under increased pressure to meet the growing demand for food at global level. Moreover, climate change will impact on crops, affecting food production; energy consumption will be critical for FP industries and the dramatic increase of the so-called "middle class" will generate a critical demand of higher hygiene and quality. The same will be for industry players, who will be asked to be compliant to stringent requirements in terms of food safety.

Specifically, a pressing growing need of high-quality food products is emerging, requiring food to be inherently equipped with its own bioactive elements even after the industrial processing phases for distribution. The only solution to this primary requirement is intelligent food-processing engineering minimizing the use of chemical additives while maximizing food integrity and environmental sustainability of the whole production and distribution workflow.

\section{Objective}

The present experimentation comes within a modernization trend of technologies related to processing of food and food materials, where Radiofrequency (RF) and Microwaves (MW) are often considered as alternative methods for treatment of agrofood products. In particular, the objective of the work was the definition of a modern, multi-feed MW processor based on solid-state generators.

Sergio Fontana $\mathrm{Srl}$ is a Company operating in the production of natural active principles, obtained by high-quality processing of food materials which are inherently rich in healthy bioactive compounds. Different processes are implemented, all sharing the essential need of maximally preserving the precious organoleptic properties of the incoming raw materials. Many promising applications of dielectric heating have been implemented in the FP industry; however, there are several potential problems that need to be addressed before the technology can be successfully applied to food in an industrial scale. One of the major issues of dielectric heating is the potential of incurring in hot spots within a non-homogeneous target. When this natural physical effect comes along with surface irregularities and/or inappropriate packaging, the final food quality can be severely damaged with serious economic loss. 
The microwave processor had to be designed as a flexible tool for disinfection of dry materials, possibly infested by insects or unwanted microorganisms accumulated during the previous stocking or delivery phases, and for accurate pre-heating of fresh materials to allow for optimal subsequent extraction processes.

The processor had to be conceived as a batch oven, equipped with a single front door for the load/unload operations and built to allow the presence of three parallel layers, each capable to carry two polypropylene trays containing the food materials to be processed.

The full operational flexibility of the oven, intended to work with both fresh raw materials and with dry herbs soon emphasized a clear design target: in the former case the microwave energy is dissipated into the food materials while in the latter case the dielectric loss should be ideally concentrated into the infesting organisms while safeguarding the precious bioactive elements contained in the dry products, which typically suffer from overtemperature stress.

The technical specifications of the microwave processor can be characterized by the following functions:

- availability of $3 \mathrm{~kW}$ MW power with possibility to differentiate the radiation amount among the three layers thanks to multiple feed;

- capability to extract water vapor when required;

- capability to be remotely programmed by means of standard web browsers from fixed or mobile terminals;

- capability to operate with fully customizable "recipes" allowing smart combinations of Continuous Wave $(\mathrm{CW})$ and pulsed modes, even with different radiation modes generated by each feed

- capability to measure in real-time the amount of reflected energy at every radiation port to allow self-regulating algorithms aiming at process optimization.

\section{The "kopernicook" modular architecture}

The required system has been designed by a modular architecture where the elementary module is a $250 \mathrm{~W} 2450 \mathrm{MHz}$ solid-state generator equipped with an embedded interface board allowing serial communication within a digital communication bus through RS485 standard.

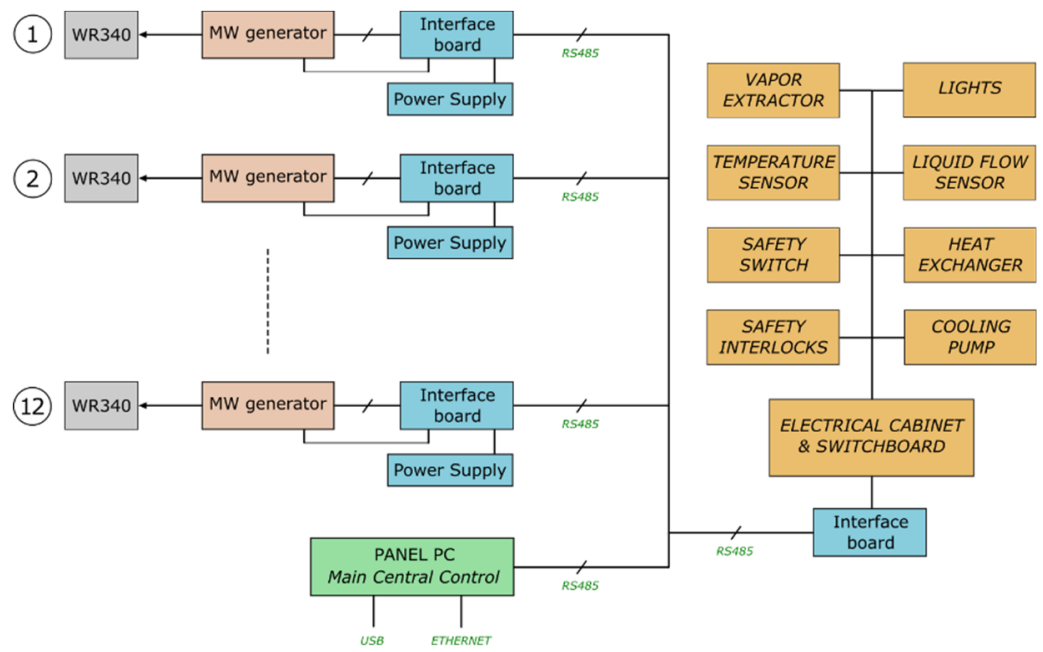

Fig. 1. Block diagram of the Kopernicook MW Processor 
The reverberating chamber, conceived to host the 6 trays distributed over the three parallel layers, has been designed with six feeds on the left wall and six on the right wall, vertically and horizontally aligned in order to have four $250 \mathrm{~W}$ feeds per each layer.

Due to the inherently multimodal behavior of the chamber, the field distribution in each layer is influenced by the contribution of each of the microwave generators and the overall field distribution also depends on the type and amount of materials contained in the trays. Moreover, due to the time-varying dielectric properties of the food materials, the field distribution also dynamically changes during the running process, determining a complex interaction with the materials that needs to be monitored and managed by the distributed control system.

Each generator is equipped by an output circulator guaranteeing safe operation even with $100 \%$ reflected power and by an autonomous microprocessor-based control system with local monitoring and management capabilities, triggering immediate protection countermeasures at the occurrence of conditions that might damage the generators hardware.
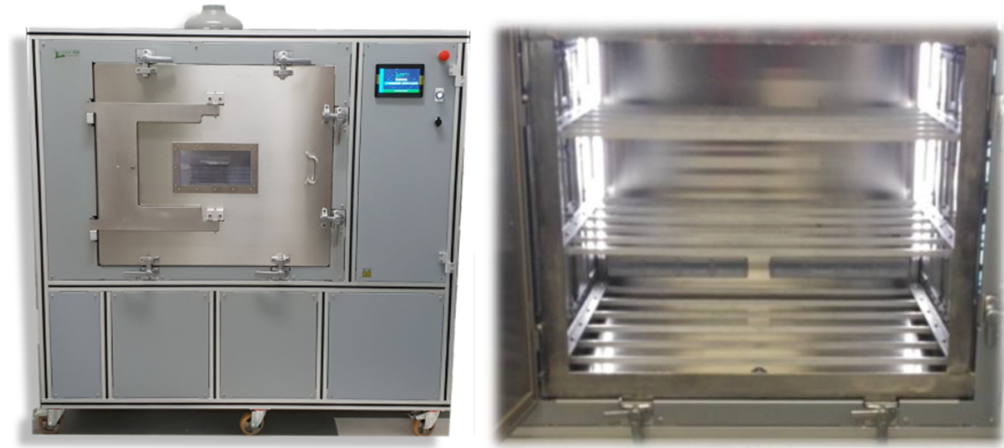

Fig. 2. Front view of the Microwave Processor and internal view of its chamber

All the operation of the microwave processor is regulated by a "Recipes Editor", a web application running on the central control unit, accessible by any web browser from remote locations. The editor allows creating "recipes" (arbitrary compositions of microwave emission phases, totally configurable by the end user) and uploading them in a dynamic database in the central control unit. The editable parameters are recipe duration, frequency, $\mathrm{CW}$ or Pulsed radiating modes, phase durations, power levels and activation/deactivation of vapor extractor, and each phase can be added in "simple" or "advanced" mode. A simple phase drives all the generators simultaneously with identical parameters, an advanced phase allows every single generator to radiate with independent parameters and both simple and advanced phases can be combined within a recipe.

The central control unit, based on an industrial-grade Panel PC, allows to select one of the uploaded recipes and to run it with the loaded materials. The software allows to monitor the operating parameters and, in particular, it shows the real-time measurement of the twelve reflected powers values.

Figure 3 shows some screenshots from the Recipes Editor application with details about parameters setting, in CW, pulsed, simple and advanced modes. 


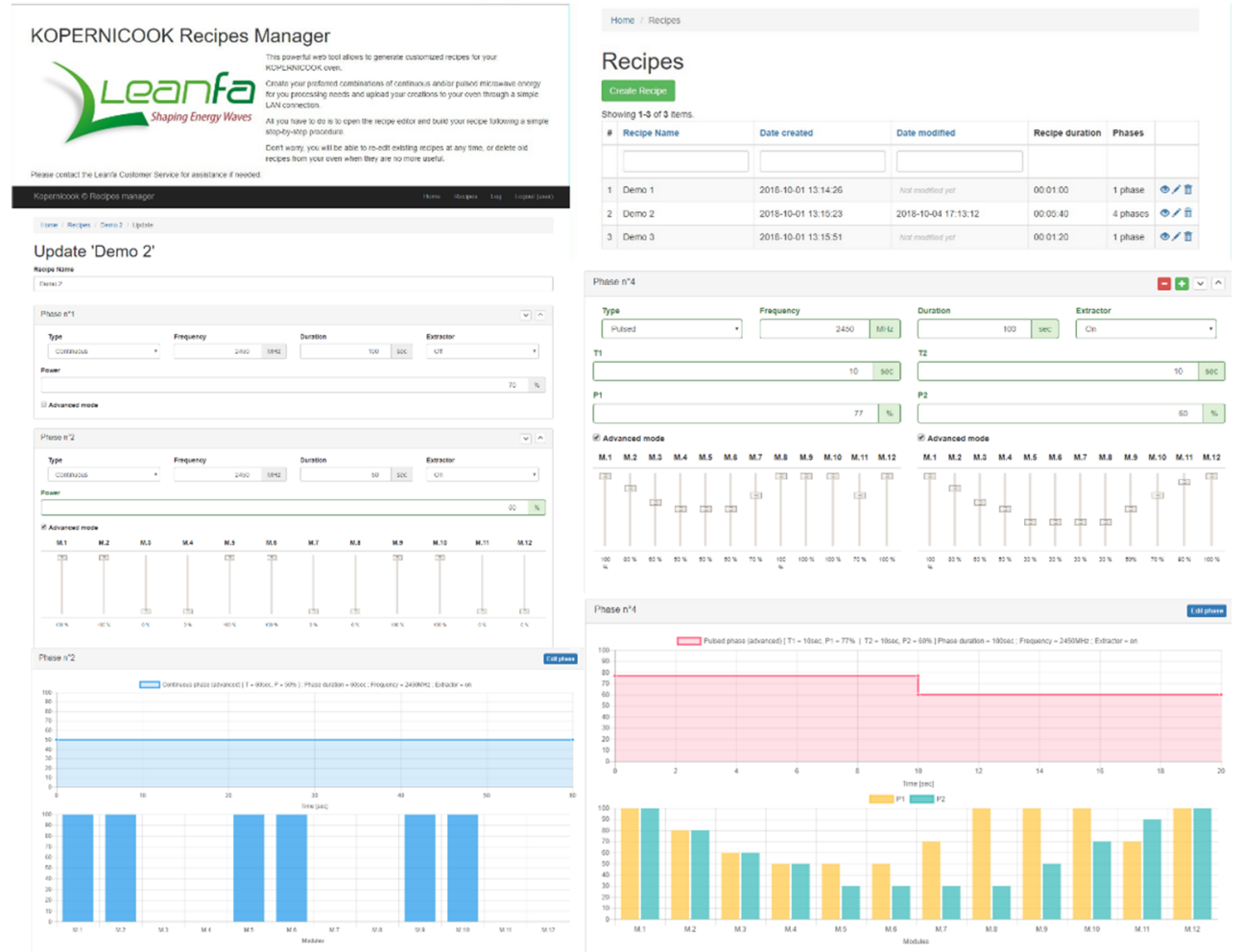

Fig. 3. Details of the Recipes Editor web application

The central control unit is not simply a collector of recipes, it is much more a high-level supervisor that, thanks to real-time monitoring of the reflected power levels at every microwave port, is capable to dynamically monitor - both in the time and space domains the capability of the materials to absorb the radiated energy and to evaluate cross-coupling contributions between the 12 ports.

This information is used to trigger a self-regulating algorithm (indicated by a brain icon on the front-panel GUI, see Fig. 4) which automatically takes control of the radiated power from each port, replacing the original recipe until certain well-tailored conditions are met.

\section{Self-regulating algorithms}

Different self-regulating algorithms have been implemented, based upon the definition, for each port, of two thresholds of reflected power level, that activate or deactivate a "foldback" power function, which is triggered by the central control system and autonomously managed by the control system of each microwave generator. Moreover, a real-time automatic selection of the optimal operating frequency (in the $2400-2500 \mathrm{MHz}$ range) has been implemented, used to achieve uniform thermal profiles with the minimum possible power consumption.

The algorithm has been designed to accomplish different goals:

- protection of the generators' hardware;

- overall minimization of the reflected energy during the process; 
- correction of deviations from the nominal expected process due to parametric shifts in the targets' physical characteristics.

The system has proven to be very robust and, thanks to the hysteresis guaranteed by userselectable reflected power thresholds, it always reaches a stable steady-state operating condition.
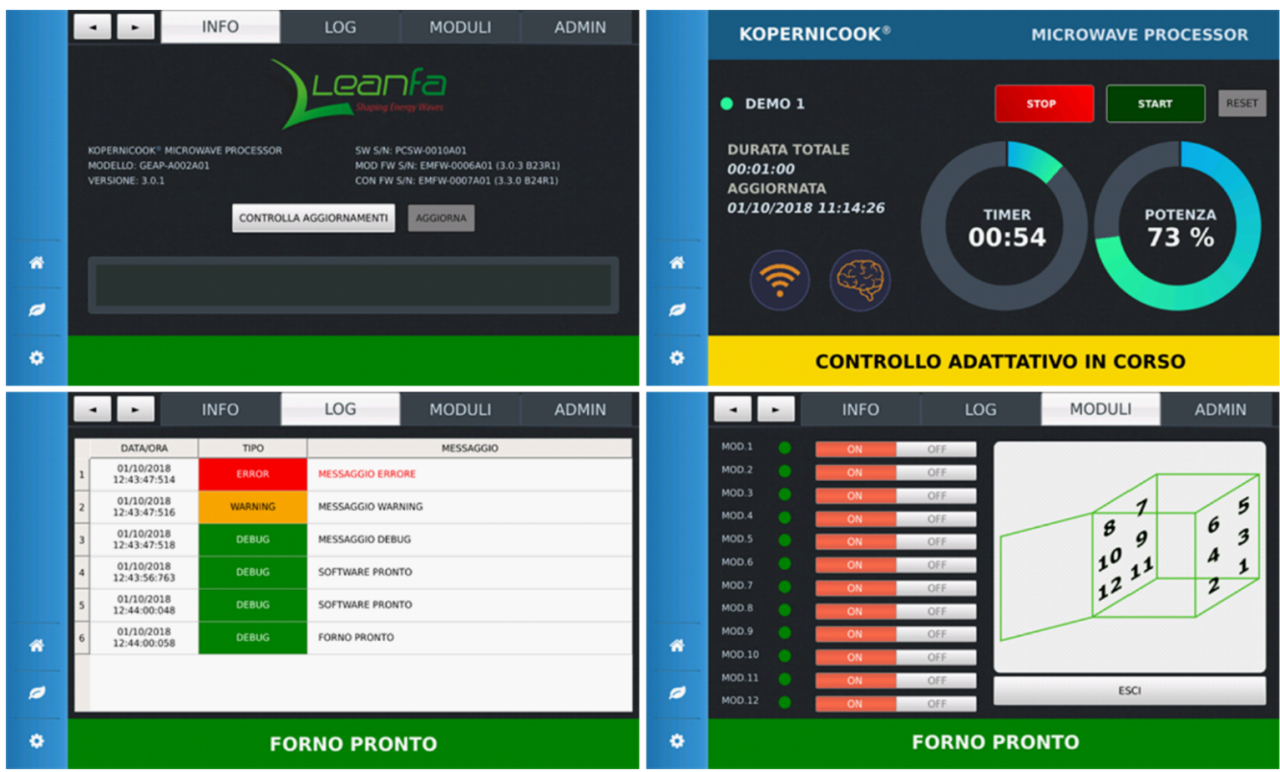

Fig. 4. Screenshots from the central control panel

The following algorithm describes one of the most effective self-regulating workflows, used with dry materials (a simplified pseudo-code is used here for schematization).

1) Central control unit:

if state $==0$

send OutPwrLev to all active gen

if (number of gen with RefPwr $\geq$ HighRefThreshold) $\leq 2$

switch off only those gen

state $=1$

else if (number of gen with RefP wr $\geq$ HighRefThreshold) $>2$

send FoldbackOn command to all active generators

state $=1$

if state $==1$

else keep state $=0$

if (number of active gen with RefPwr $>$ LowRefThreshold) $==0$

send FoldbackOff command to all running gen

state $=0$

else keep state $=1$

2) Each active generator unit in the running recipe:

if state $==0$

set $F w d P w r=O u t P w r L e v$

keep state $=0$ 


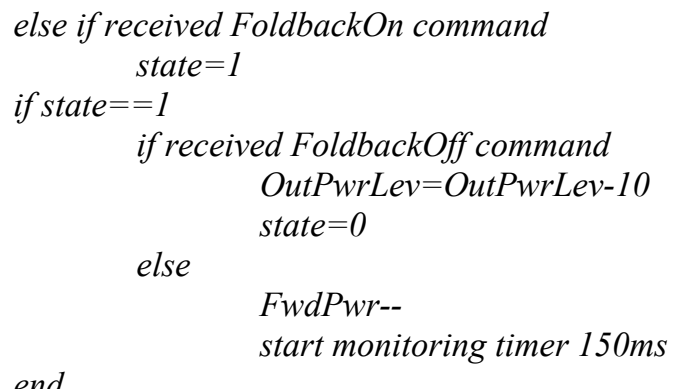

The algorithm is designed to manage the overall field distribution with a distributed intelligence approach by taking control of the process until the end of each phase in a running recipe is reached. At the beginning of the following phase in the same recipe, the control is again taken by the central control unit obeying to the nominal recipe.

\section{Results}

When enabled, the self-regulating algorithm works by automatically reducing the overall radiated power to a safe level corresponding to the user-defined threshold and later trying to recover a higher radiating level, slightly lower than the previous nominal level. The result is an optimal convergence towards minimum reflected energy, smoothly following the natural tendency of the materials to absorb less microwave power while their temperature increases and their water content decreases. After a progressive fine tuning of the recipes conceived by the engineers of Sergio Fontana Srl, the self-regulating process triggers only when the loaded materials show stronger deviations from the nominal range of physical parameters as weight, moisture and/or initial temperature.

Lower deviations (less than 10\%) have been observed on the overall radiated power with fresh materials, while higher deviations (up to $28 \%$ ) have been observed when operating with dry herbs, in accordance with the well-known sensitivity of the dielectric properties to the average moisture level.

Moreover, the distributed intelligence approach has demonstrated to allow even better regulation in case of parametric deviations limited to one or two layers in the processing chamber, allowing automatic power level differentiation according to the different capability of each layer to absorb the radiated energy.

In the following Figure 5 three different sample thermal profiles are shown, obtained with different recipes applied to fresh fruit (left and center) and to dry herbs (right).
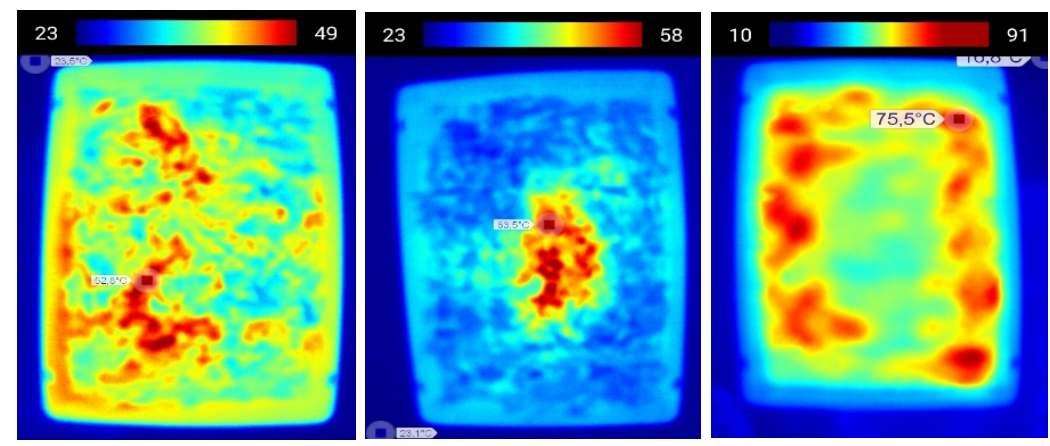

Fig. 5. Thermal profiles obtained with different fine-tuned recipes 
In particular, in-field operation has demonstrated the usefulness of pulsed radiation modes for disinfection of dry herbs, helping in concentrating the dielectric loss into the infesting organisms, while avoiding injuries on properties of the food materials generated by unwanted overtemperatures. The most homogeneous thermal results, correlated to higher disinfection rate, have been achieved thanks to the application of frequency shift using recipes with a combination of $\mathrm{CW}$ radiation modes during the first 5-7 minutes of operation and pulsed modes with decreasing duty cycles during the following 15-23 minutes, enabling water vapor extraction during the initial $30-40 \%$ of the recipe.

\section{Conclusions}

The complete absence of mechanical stirring elements in the microwave processor is compensated by the distributed architecture with 12 solid-state generators, each fully configurable in terms of radiating parameters by means of the Recipe Editor application. By using well-tailored recipes, it is possible to obtain fairly well controlled thermal profiles over the material distributed on the three layers.

Further development will be performed by progressively moving to radiation algorithms with arbitrary waveforms and by adding real-time temperature and moisture sensors to fulfil a complete monitoring network for a more accurate generation of feedback to the distributed control architecture. The technological roadmap pictured by power devices manufacturers is further encouraging new development effort into accurate hardware-software co-design aimed at facilitating the desired industrial scale-up towards efficient continuous processing systems.

\section{References}

1. OECD, "Population and growth rates, 1950-2050, actual and projected", in Local economic strategies and systems, OECD Publishing, Paris, 2014

2. Yakovlev, V. V., IEEE MTT-S International Microwave Symposium, 2015

3. Tiefeng Shi, \& Kaldi Li., IEEE/MTT-S International Microwave Symposium Digest, 2012 (generatore 157W CW 2450MHz)

4. Piyasena, P., Dussault, C. Koutchma, T., Ramaswamy, H.S., and Awuah, G.B., Critical Reviews in Food Science and Nutrition 43(6), 587-606, 2003

5. Zuber, S., Joss, M., Tresch, S., Kleingries, M., 21st International Drying Symposium, Valencia, 2018

6. Przemyslaw Korpas, Mateusz Krysicki, and Andrzej Więckowski, Multiphysics Models and Materials properties, Karlsruhe, Germany 2014

7. Awuah, G., Ramaswamy, H., \& Tang, J., Radio-Frequency Heating in Food Processing, 323-353, 2015 